\title{
Direct Counting Analysis on Network Generated by Discrete Dynamics
}

\author{
Shu-Hao Liou* and Chia-Chu Chen ${ }^{\dagger}$ \\ Department of Physics, National Cheng Kung University, Tainan, Taiwan 70101
}

(Dated: August 28, 2018)

\begin{abstract}
A detail study on the In-degree Distribution (ID) of Cellular Automata is obtained by exact enumeration. The results indicate large deviation from multiscaling and classification according to ID are discussed. We further augment the transfer matrix as such the distributions for more complicated rules are obtained. Dependence of In-degree Distribution on the lattice size have also been found for some rules including $\mathbf{R} 50$ and $\mathbf{R} 77$.
\end{abstract}

PACS numbers: 89.75.Hc, 89.75. Da, 89.75.Fb.

Discrete dynamical system in general can generate very complicated dynamics even though the rule or the equation of evolution for the system can be apparently simple. Examples include the synchronization of Pulse-coupled Oscillators, chaos arises from discrete mapping and more interestingly the simple Cellular Automata (CA) introduced by von Neumann almost half a century ago [1]. One of the important problems in discrete dynamical system is the classification of it's general behavior. Generally speaking this is a hard question to be answered since the classification requests a full understanding of the dynamics itself. Even for simple system such as CA which evolves according to simple rules, a complete classification is still controversial since the work of StevenWolfram in the 80' of last century [2]. Recently, A. Shreim et al. have approached the CA problem by employing concepts originated from network analysis [3]. They classified CA by analyzing the local and global properties of the network generated by the dynamics of CA. Their approach certainly provides new way to address this interesting and difficult problem. In this work we follow up their approach and investigate further on this problem. Here we would only concentrate on the local analysis and will return on the global property later.

For any discrete dynamical system, the configuration space can be represented by discrete nodes. The time evolution of the system in each time step is presented by a connected link starting from one node to its dynamical successor. As a result, all the trajectories of the dynamical system generated a directed network. Since CA are deterministic systems, each node possess one single outgoing link. However, due to the existence of fixed points and periodic solutions, the dynamics of CA in general is irreversible and the number of preimages of a state can be larger than 1 . The in-degree (ID) of a node is defined as it's number of preimages and obviously in CA the in-degree is a local property. In [3] the authors have performed analysis on the ID distribution for various one dimensional CA both analytically and numerically. Due to the simplicity of Rule 4 (R4), by using a transfer matrix (T) approach together with combinatory analysis on preimages, they obtained an approximation solution of the probability distribution $P(k)$ with $k$ denoting the indegree. Their results were presented on the $\log -\log$ plot as curves instead of straight lines and seem to suggest the existence of multiscaling distribution. In our work, instead of using approximation, we re-analyze this problem by performing direct counting of the in-degree for various rules with different lattice size $L$. What we have found is that the distribution is not as simple as what have been obtained in 3]. The plan of this report is as follows. A brief summarization on nomenclatures of CA and network are given and the results on ID of [3] are briefly discussed in section [ The direct counting results of various rules are reported in section [1] where some further studies on the ID are also treated. The extension of transfer matrix for more accurate analysis on other rules and its implication are treated in Section III. A brief summary of our findings is provided in the final section.

\section{INTRODUCTION}

A one-dimensional Cellular Automaton is defined on a spatial lattice of $L$ sites where $L$ can either be finite or infinite. In this work we will keep $L$ finite and the state of each site is in one of the $g$ states at any time $t$. Each site follows the same prescribed rules for updating. For elementary CA, the number of neighborhood of each site is 2. The state of CA starts out with arbitrary initial configuration which is represented by $S(0)=\left\{s_{0}, s_{1}, s_{2}, \ldots, s_{L}\right\}$ where $s_{i}$ can be in any one of the $g$ states. The configuration of system at time $t$ is denoted by $S(t)=\left\{s_{0}^{t}, s_{1}^{t}, s_{2}^{t}, \ldots, s_{L}^{t}\right\}$. In this work the state of each site is restricted to $g=2$ such that $s_{i} \in\{0,1\}$. Let $\mathbf{R}$ be any one of the $\mathrm{CA}$, the value of $s_{i}$ at $t+1$ time step is set equal to $R\left(s_{i-1}^{t} s_{i}^{t} s_{i+1}^{t}\right)$ which is equal to 0 or 1 according to the rule of interest. Apparently for elementary CA there are 256 rules. Following Wolfram's notation each rule can be assigned with a number given by:

$$
\begin{aligned}
R(000)+2 R(001) & +2^{2} R(010)+2^{3} R(011)+2^{4} R(100) \\
& +2^{5} R(101)+2^{6} R(110)+2^{7} R(111) .
\end{aligned}
$$

For example, Rule $237(\mathbf{R} 237)$ is the one with $R(100)=$ $0, R(001)=0$ and all others $R\left(s_{1} s_{2} s_{3}\right)=1$. Based on large number of numerical studies, Wolfram has suggested that CA can be classified into four classes [2]. Cellular Automata within each class have the same qualitative behavior. Starting from almost all initial conditions, 
trajectories of CA become concentrated onto attractors, the four classes can then be characterized by their attractors. According to Wolfram's classification, classes I, II and III are roughly corresponding to the limit points, limit cycles and chaotic attractors in continuous dynamical systems respectively. More precisely their respective long time limits are: (I) spatially homogeneous state, (II) fixed (steady) or periodic structure and (III) chaotic pattern throughout space. The fourth class of CA behaves in a much more complicated manner and was conjectured by Wolfram as capable for performing universal computation. For finite one-dimensional CA, the lattice is arranged on a circle with periodic boundary conditions. Such Cellular Automata have a finite number of states $N=2^{L}$, and as a result, after sufficient long time evolution the system must enter the state which can either be homogenous, steady or periodic state. Therefore, the class III and IV attractors do not exist in finite CA. However, it is also known that classes III and IV are hard to distinguished in some cases and resulting with disputed classification. In some sense this is due to the lack of a sensible way for defining complexity which is the criterion for class IV. In [3] A. Shreim et al. studied networks generated by $\mathrm{CA}$, they claimed to have found highly heterogeneous state space networks for classes III and IV in contrast to the networks generated by classes I and II. One of the characteristic of state space is the in-degree distribution $P(k)$ which is defined as [3]

$$
P(k)=\frac{\Sigma}{N} .
$$

where $L$ is the size of the lattice, $N=2^{L}$ is the total number of state and $\Sigma$ is the number of state with in-degree $k$. However in their analysis on the local properties, approximation has been applied in the analytical calculation of ID which shows nontrivial scaling. For completeness we briefly summarize their method of calculation in this section. They introduced the transfer matrix $\mathbf{T}$ which maps each pair $s_{i-1} s_{i}$ onto the pair $s_{i} s_{i+1}$, and such $\mathbf{T}^{(s)}$ could be used to characterize all the preimages of hubstates (A hubstate is the state which has the maximum in-degree $k_{m}$.) The rows and columns are order as " $00 "$, " $01 "$, " 10 " and " 11 " accordingly. For example the $\mathbf{T}$ matrix of $\mathbf{R} 4$ is given by:

$$
\mathbf{T}_{4}=\left(\begin{array}{llll}
1 & 1 & 0 & 0 \\
0 & 0 & 0 & 1 \\
1 & 1 & 0 & 0 \\
0 & 0 & 1 & 1
\end{array}\right)
$$

It is easy to check that the maximum in-degree $k_{m}$ is related to $\mathbf{T}$ as

$$
k_{m}=\operatorname{Tr}\left(\mathbf{T}^{L}\right)
$$

The scaling of $k_{m}$ with the lattice size can be obtained by using the largest eigenvalue $\lambda_{m}$ of $T$, namely, $k_{m} \approx N^{\nu}$ with $\nu=\log _{2} \lambda_{m}$. Furthermore, for $\mathbf{R} 4$ we assigned $n$ as the number of isolated $1^{\prime} s$, for example two configurations (010000) and (010100) have $n=1$ and 2 respectively. Then by assuming a one-one corresponding relation between $k$ and $n$, the following expression was proposed for the in-degree distribution:

$$
P(k) d k=\frac{\Omega(n) d n}{2^{L}} .
$$

A multiscaling result is then obtained and given by the following expression [3] :

$$
y=-1-x+\log _{2}\left[\frac{(1-\epsilon)^{1-\epsilon}}{\epsilon^{\epsilon}(1-2 \epsilon)^{1-2 \epsilon}}\right] .
$$

where $y=\log P(k) / \log N, x=\log k / \log N$ and $\epsilon=n / L$. The result of Eq. (5) was ploted in [3] and are apparent curvature appeared on the curve as such a conclusion of nultiscaling method than finite-size effect was proposed. Similar results of multiscaling in other rules were also reported in their article by using the same approximation approach. To compare the results of [3] with exact calculation we have performed the exact enumeration of $\mathbf{R} 4$ with different $L$. For the lack of space, in Table I we only list all the in-degree's for $L=12$. The same characteristic also exist for other values of $L$. One notices that for the same $k$ there corresponds more than one value of $n$. For example, for $k=12, n$ can either be 2 or 3 . Therefore the meaning of $d n / d k$ is ambiguous. One might argue that the multi-valueness of $n$ is an artifact of small $L$. However we have checked with $L=10^{4}$ where different states with the same $k$ were found. In fact, for $k=1$, states of different $n$ can be constructed easily. For example, for $k=1$, we have found at least 2 states $(000100010001 \ldots)$ and $(010101 \ldots)$ which correspond to $n=2500$ and 5000 respectively. Since the results were obtained for small $k$, there is a possibility that $\mathrm{dn} / \mathrm{dk}$ might be well-defined for large $k$. To refute this reasoning, we have performed the large $k$ analysis for different $L$. For $L=12, k_{m}=852$ with $n=0$ and the second maximum in-degree $k_{2 m}=114$ with $n=1$; even in $L=25$, we got $k_{m}=1276941$ with $n=0$ and $k_{2 m}=170625$ with $n=1$. The results indicate that for $\triangle n=1$ the $\triangle k=k_{m}-k_{2 m}$ is not a small quantity. In fact $\triangle k$ rises exponentially as $L$ getting larger. As a result, $d k$ can never be infinitesimal implying the meaningless of $d n / d k$. Thus the simple multiscaling results in [3] is in doubt.

The result of Eq. (4) is presented by the solid line in Figure 1] By substituting the corresponding $k_{m}$ in Eq. (5), one has $y=-1.8$. For $\mathbf{R} 4$ with $L=25$, the results of $n-k$ relation are provided in Figure 11. There is only one hubstate with $n=0$ in such system, consequently the exact $P\left(k_{m}\right)$ equals to $1 / 2^{L}$ [4] which implies $y=-1$ independent of $L$ instead of -1.8 as obtained by the approximation calculation. Obviously the approximation for getting Eq. (5) is inadequate. In fact the above discrepancies prompted us to re-analyze this problem. 
TABLE I. The relation of in-degree $k$ and $n$ for $\mathbf{R} 4$ with $L=12$.

\begin{tabular}{ccc|ccc}
\hline \hline$k$ & $n$ & $\begin{array}{c}\text { number of } \\
\text { states }\end{array}$ & $k$ & $n$ & $\begin{array}{c}\text { number of } \\
\text { states }\end{array}$ \\
\hline 852 & 0 & 1 & 7 & 3 & 24 \\
114 & 1 & 12 & 4 & 3 & 48 \\
37 & 2 & 12 & & 4 & 12 \\
21 & 2 & 12 & 2 & 3 & 24 \\
16 & 2 & 6 & & 4 & 3 \\
14 & 2 & 12 & 1 & 3 & 4 \\
14 & 2 & 12 & & 4 & 57 \\
12 & 2 & 12 & & 5 & 36 \\
& 3 & 12 & & 6 & 2 \\
\hline \hline
\end{tabular}

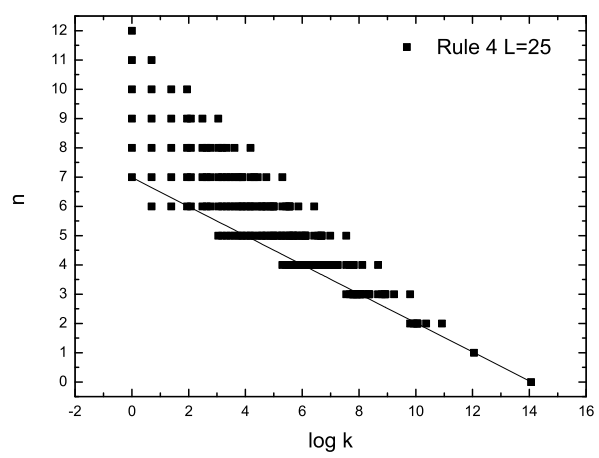

FIG. 1. The relation of $n$ and in-degree $k$ for Rule 4 . The solid curve represents Eq. (6) in [3]].

\section{NETWORK CHARACTERISTICS BY DIRECT ENUMERATION}

As suggested in [3], local properties such as the scaling of maximum in-degree with lattice size and the in-degree distribution might be useful for characterizing networks and hopefully also provide some help to classify CA. However, from previous discussions it is clear that approximation calculations can lead to inadequate conclusions such as multiscaling of ID being an example. In order to have a better understanding on this kind of network, it is unavoidable to address the problem with exact enumeration which will be the approach adopted in this work. We have studied more rules from different classes suggested by Wolfram [2] and the in-degree distributions are evaluated exaclty by direct counting method. The transfer matrix approach proposed in [3] has been used in this work extensively.

It is important to note at the outset that not all rules can have a transfer matrix representation. The following matrix represents possible candidate of $\mathbf{T}$ for elementary
TABLE II. The classification for different rules with $\lambda_{m}$.

\begin{tabular}{lll}
\hline \hline$\lambda_{m}$ & $\nu$ & Rules \\
\hline 1.32472 & 0.4056 & 110,62 \\
1.46557 & 0.551 & $232,33,6$ \\
1.61803 & 0.6942 & $36,18,5$ \\
1.75488 & 0.811 & 4,32 \\
2 & 1 & 0,255 \\
\hline \hline
\end{tabular}

CA:

$$
\mathbf{T}=\left(\begin{array}{cccc}
a_{1} & a_{2} & 0 & 0 \\
0 & 0 & a_{3} & a_{4} \\
a_{5} & a_{6} & 0 & 0 \\
0 & 0 & a_{7} & a_{8}
\end{array}\right)
$$

where $a_{i}, i=1 \sim 8$, can take on either 1 or 0 . In fact there are totally 256 different matrices represented by $\mathbf{T}$, however it is also known that not all of them can represent the 256 elementary $\mathrm{CA}$. This is partly due to the fact that not all rules can have a $\mathbf{T}$ matrix representation. In particular, by diagonalizing all possible $\mathbf{T}$, we have obtained only 9 different $\lambda_{m}: 1,1.22074,1.32472$, $1.38028,1.46557,1.61803,1.75488,1.83929$ and 2. Some of the rules corresponding to different $\lambda_{m}$ are listed in Table II.

In what follows, all ID are evaluated by direct counting. To set the stage we present the in-degree distributions of various rules with different $L$ in Fig. 2, the results of $\mathbf{R} 4, \mathbf{R} 22$ and $\mathbf{R} 110$ are plotted. The results of each rule seem to show the same distribution for three different values of $L$. Especially for small $k$ the distributions are in good agreement for different $L$ with all the points fall on a simple curve with non-vanishing curvature. As $k$ increases, the distribution becomes more spread out but still maintains the same distribution for various $L$. These observation seems to suggest that the ID of this kind of network is scale independent. Moreover, since these rules belongs to different classes and the scale independent of the distributions seem to be universal for all four classes of CA. Similar results can also be obtained for other rules and will not be shown explicitly. Even though the $L$ independence of ID is very suggestive, however a definite answer would require a full scale analysis with larger lattice size. Work along this line is now being studied and will be reported separately. On a different front, with knowing all the possible values of $\lambda_{m}$, it is natural to wonder if rules with the same $\lambda_{m}$ are correlated. To show whether such correlation exists or not, results of the same $\nu=\log _{2} \lambda_{m}$ are plotted in Fig. 3 and 4. For $\nu=0.4056$, the ID of R193, R147, $\mathbf{R} 110, \mathbf{R} 137, \mathbf{R} 124$ and $\mathbf{R} 54$ are given in Fig. 3, all of which show similar distribution. Interestingly these rules belong to the same class, namely class four according to Wolfram's scheme. For clarity, in Fig. 4 we have also plotted the ID of other rules of class IV. They also share the same feature as given in Fig. 3. Similarly, the result for other are given in Fig. 5)(a) with $\nu=0.551$, the 
corresponding rules are all of class II [5] and the ID of each rule also shows a universal distribution. However such good correlation between ID and $\nu$ is not conclusive. Furthermore rules from different classes can have the same $\nu$. For example, the plot given in Fig. 5(b) for the in-degree distributions of various rules corresponding to $\nu=0.6942$ shows diverse patterns. It is noted that in this plot, R36 and R5 are class II, whereas R18 and R126 are classified as class III [5]. To make things worse, the plot for various rules of class II is given in Fig. 5(c), where apparently the patterns get more diverse. More importantly, those rules belong to different $\nu$ 's. As a result, one might conclude that there are good correlation for class IV which possess $\nu=0.4056$, apart from that the ID of other classes do not share a universal pattern and also the value of $\nu$ can not be used as a indicator for classification. Thus the local properties such as $\nu$ and in-degree distribution are not useful for CA classification as suggested in [3].

\section{AUGMENTATION OF T MATRIX}

The $\mathbf{T}$ matrix approach for calculating maximum indegree is quite efficient and there are also cases in which the in-degree can also be evaluated exactly. However, as clearly stated in the previous section that $4 \times 4 \mathbf{T}$ matrix do not always exist for elementary CA and therefore it is interesting to see how modification on the transfer natrix scheme can be extended to other rules. In this section an extension is suggested by augmenting the $4 \times 4$ matrix to a $8 \times 8$ matrix. Instead of mapping each pair $s_{i-1}^{t} s_{i}^{t}$ onto the pair $s_{i-1}^{t} s_{i}^{t}$, $\widetilde{\mathbf{T}}$ will map $s_{i-2}^{t} s_{i-1}^{t} s_{i}^{t}$ into the triplet $s_{i-1}^{t} s_{i}^{t} s_{i+1}^{t}$ and hence define a $8 \times 8 \widetilde{\mathbf{T}}$ matrix which could be used to describe the preimage more efficiently. The basis states are

$|i\rangle \in\{(111),(110),(101),(100),(011),(010),(001),(000)\}$.

Fig. [6] shows the definition of $8 \times 8 \widetilde{\mathbf{T}}$ matrix.

There are some merits in this approach. First of all, for elementary CA where all the rules of evolution involve three adjacent cells, obviously such extension certainly respect the evolution dynamics and hence include more information of the dynamical system. Secondly, the augmentation of $\mathbf{T}$ indeed resolves some of the cases which can not be done with $4 \times 4$ matrices. Moreover, this approach can also be applied to other more complicate CA including the model with next nearest-neighbor interaction, we will illustrate that by an example in this section.

It is easy to see that whenever the $4 \times 4$ transfer matrix can be defined then there is always a corresponding $8 \times 8$ matrix however the reverse is not always true. For the case when both descriptions exist then a natural question about the uniqueness of the results arises, namely, can both $4 \times 4$ and $8 \times 8$ matrices provide the same results? This is due to the fact that the eigenvalues of both
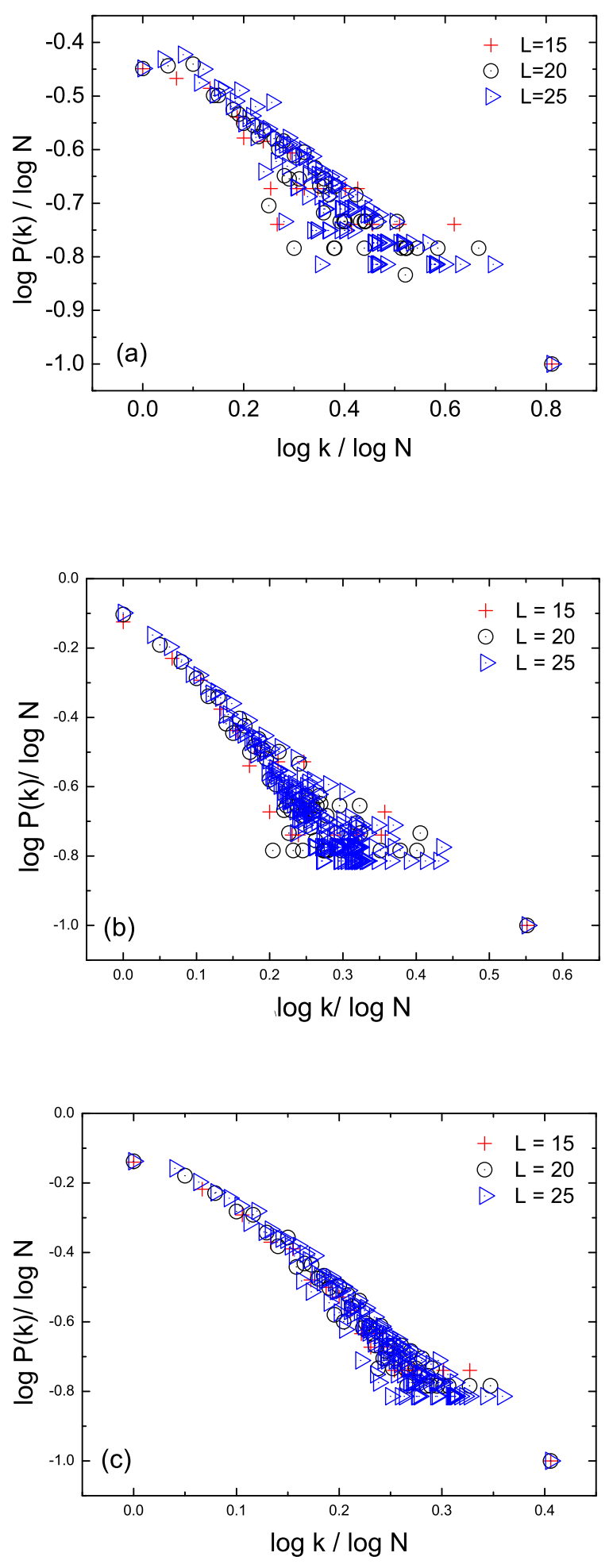

FIG. 2. The direct counting results of ID for (a)R4, (b)R22 and (c)R110 with different $L$. 

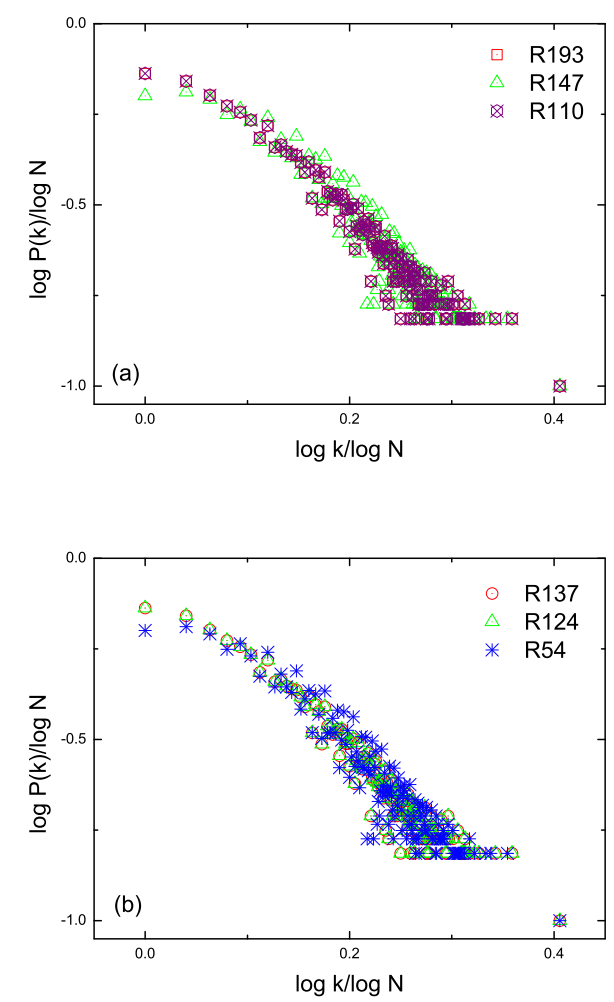

FIG. 3. The In-degree Distributions for (a) R193, R147, R110 and (b) R137, R124 and R54 with $L=25$.

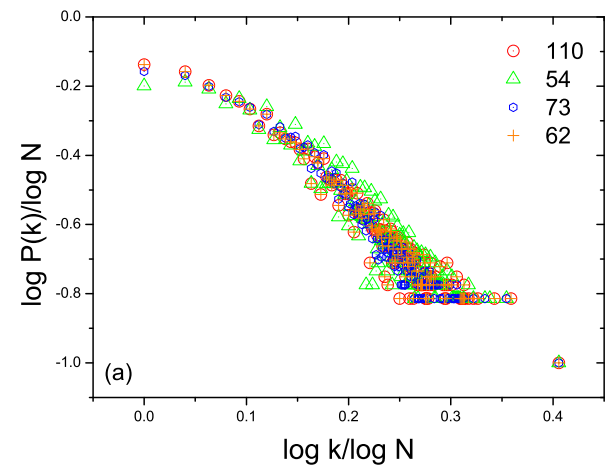

FIG. 4. The In-degree Distributions for R110, R54, R73 and R62 with $L=25$.

matrices can be very much different and there is no obvious reason for them to be the same. For all the cases we have studied the maximum in-degree can be exactly calculated by both $4 \times 4$ and $8 \times 8$ matrices and the results are identical. Unfortunately there exists no analytical proof. To illustrate this fact, the following example is the calculation of $k_{m}$ with the $\widetilde{\mathbf{T}}$ matrices for $\mathbf{R}_{18}$. Fig. 7 is the evolution rule of $\mathbf{R}_{18}$, and the $4 \times 4 \mathbf{T}$ matrix
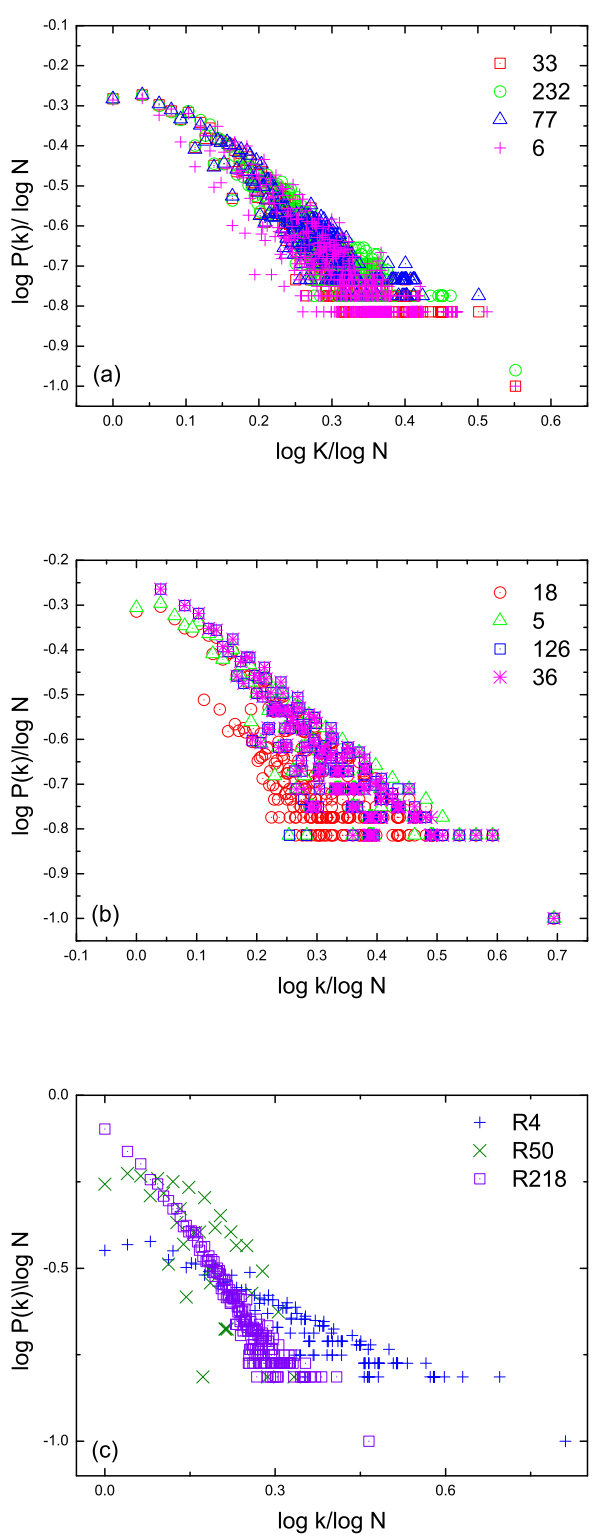

FIG. 5. The in-degree distributions for various rules with (a) $\nu=0.551$ and (b) $\nu=0.6942$; (c) The in-degree distributions for Class II, where $\nu=0.811$ for $\mathbf{R} 4, \nu=0.3471$ for $\mathbf{R} 50$ and $\nu=0.4650$ for $\mathbf{R} 218$. All of results are ploted with $L=25$.

obtained in Amer Shreim et al.'s study [3] is:

$$
\mathbf{T}_{18}=\left(\begin{array}{llll}
1 & 0 & 0 & 0 \\
0 & 0 & 1 & 1 \\
0 & 1 & 0 & 0 \\
0 & 0 & 1 & 1
\end{array}\right)
$$

The hubstate of $\mathbf{R} 18$ is $(00 \ldots 00)$, then Fig 7 shows that (001) and (100) are excluded from the pre-images 


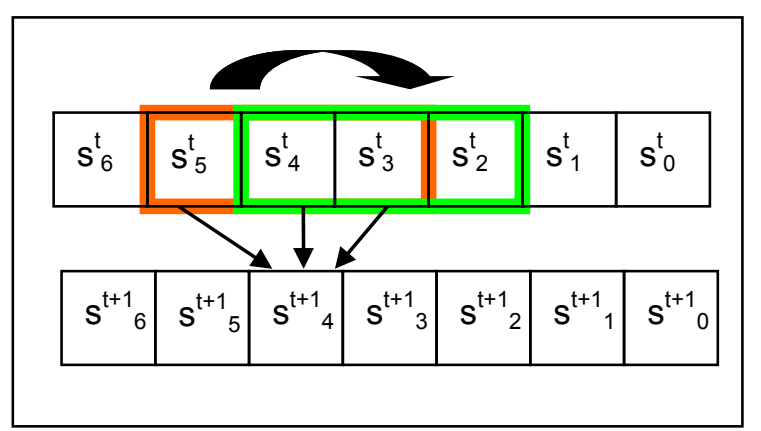

FIG. 6. The diagram for $8 \times 8 \widetilde{\mathbf{T}}$ matrix.

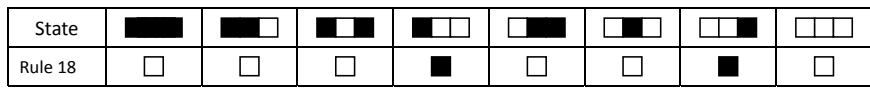

FIG. 7. The diagram of $\mathbf{R} 18$.

of (00..00), therefore one has $\widetilde{\mathbf{T}}_{18}$ :

$$
\widetilde{\mathbf{T}}_{18}=\left(\begin{array}{cccccccc}
1 & 0 & 0 & 0 & 0 & 0 & 0 & 0 \\
0 & 0 & 0 & 0 & 0 & 0 & 0 & 0 \\
0 & 0 & 0 & 0 & 0 & 1 & 0 & 0 \\
0 & 0 & 0 & 0 & 0 & 0 & 1 & 1 \\
0 & 0 & 0 & 0 & 0 & 0 & 0 & 0 \\
0 & 0 & 1 & 1 & 0 & 0 & 0 & 0 \\
0 & 0 & 0 & 0 & 0 & 1 & 0 & 0 \\
0 & 0 & 0 & 0 & 0 & 0 & 1 & 1
\end{array}\right)
$$

The in-degree of hubstate is given by $k(S)=\operatorname{Tr}\left(T^{L}\right)$, with $L=10$ one has:

$$
\mathbf{T}_{18}^{10}=\left(\begin{array}{cccc}
1 & 0 & 0 & 0 \\
0 & 34 & 55 & 55 \\
0 & 21 & 34 & 34 \\
0 & 34 & 55 & 55
\end{array}\right)
$$

and

$$
\widetilde{\mathbf{T}}_{18}^{10}=\left(\begin{array}{cccccccc}
1 & 0 & 0 & 0 & 0 & 0 & 0 & 0 \\
0 & 0 & 0 & 0 & 0 & 0 & 0 & 0 \\
0 & 0 & 13 & 13 & 0 & 21 & 21 & 21 \\
0 & 0 & 21 & 21 & 0 & 34 & 34 & 34 \\
0 & 0 & 0 & 0 & 0 & 0 & 0 & 0 \\
0 & 0 & 21 & 21 & 0 & 34 & 34 & 34 \\
0 & 0 & 13 & 13 & 0 & 21 & 21 & 21 \\
0 & 0 & 21 & 21 & 0 & 34 & 34 & 34
\end{array}\right)
$$

It is clear that the traces of these two matrices are the same and resulting the same value for the maximum indegree $k_{m}$.

We have also obtained the $8 \times 8 \widetilde{\mathbf{T}}$ matrix for more than 22 rules and also rules related to them by symmetry( by exchanging $0 \leftrightarrow 1$ ). They are: $1,2,4,5,6,7$, $18,19,22,23,33,36,40,54,62,73,94,110,160,218$ and 232. Except for $\mathbf{R} 94$, all these rules possess both $\mathbf{T}$ and $\widetilde{\mathbf{T}}$ which results with the same $k_{m}$. Further application of this augmentation procedure is illustrated by
TABLE III. The configuration of hubstates in $\mathbf{R} 43$ with different $L$.

\begin{tabular}{cccc}
\hline \hline$L$ & $k_{m}$ & $\begin{array}{c}\text { one of the } \\
\text { hubstates }\end{array}$ & $\begin{array}{c}\text { number of } \\
\text { hubstates }\end{array}$ \\
\hline 10 & 5 & 0000110011 & 40 \\
11 & 8 & 00100110100 & 22 \\
12 & 18 & 001100110100 & 4 \\
13 & 13 & 0001100110100 & 26 \\
14 & 13 & 00001100110100 & 54 \\
\hline \hline
\end{tabular}

the example of the majority rule with more neighboring cells included in the evolution. The evolution rule is $R\left(s_{i-2}^{t} s_{i-1}^{t} s_{i}^{t} s_{i+1}^{t} s_{i+2}^{t}\right)=s_{i}^{t+1}$ where

$$
s_{i}^{t+1}=\left\{\begin{array}{cc}
1 & q_{i}^{t}>0 \\
0 & q_{i}^{t}<0 \\
s_{i}^{t} & q_{i}^{t}=0
\end{array},\right.
$$

and $q_{i}^{t}=\sum_{\mu=-2}^{\mu=2} s_{i+\mu}^{t}$. The $\mathbf{T}$ matrix for this rule is a $32 \times 32$ matrix and the maximum in-degree $k_{m}$ is 1414 for $L=15$. From this example is is also apparent that, in general for the evolution rule involves $2 n$ neighboring cells, the basic $\mathbf{T}$ matrix should be defined by $2^{2 n+1} \times$ $2^{2 n+1}$ matrix.

From the above discussion, one can see that with $8 \times 8$ $\widetilde{\mathbf{T}}$ matrix, more rules can be treated within this approach. As a consequence, such extension of $\mathbf{T}$ into larger matrix seems to provide a better description for network analysis. Unfortunately such approach can still not be able to cover all elementary CA. There exists rules in which the configurations of hubstates vary according to $L$ being even or odd, such as R50 and R77. On the other hand, there are also cases in which $\mathbf{T}$ varies with $L$ indefinitely, one of the good examples is $\mathbf{R} 43$. We had analyzed $\mathbf{R} 43$ with different $L$ and the result is listed in Table III The number of hubstates will change with $L$, and the period of the attractors is large, therefore it is not appropriate to calculate $k_{m}$ by using $\mathbf{T}$ matrix. For these situations, there is no scale of $k_{m}$ with arbritrary $L$.

For rules with the above irregularity special treatment is required for each particular case. In the following we will discuss R50 and R77 for illustration of their complication. The maximum in-degree of $\mathbf{R} 50$ and $\mathbf{R} 77$ can be properly described by $8 \times 8 \widetilde{\mathbf{T}}$ matrices only when $L$ is even, since the in-degree distributions will not depend with lattice length $L$.

Fig. 8 shows the evolution according to R50, and Table IV] shows the relation between maximum in-degree $k_{m}$ and lattice length $L$. The hubstate of R50 is either $(0101 \ldots 0101)$ or $(1010 \ldots 1010)$ when $L$ is even.The $k_{m}$ of $\mathbf{R} 50$ can be evaluated by $k\left(S_{L=\text { even }}\right)=1 / 2 \operatorname{Tr}\left[\widetilde{\mathbf{T}}_{50}^{L}\right]$, the factor $1 / 2$ is due to the fact that the $8 \times 8 \widetilde{\mathbf{T}}$ matrix carries the information of all preimages of both hubstates,

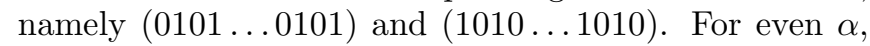
all the preimages of $L=\alpha+1$ can be obtained by just appending " 0 " or " 1 " at the boundary to all the preimages of $L=\alpha$. As a consequence, both even $L=\alpha$ and 


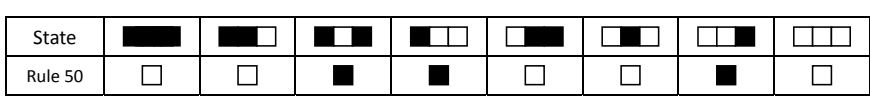

FIG. 8. The diagram of $\mathbf{R} 50$.

TABLE IV. Maximum in-degree corresponding to lattice length $L$ in $\mathbf{R} 50$ by direct counting.

\begin{tabular}{ccc|ccc}
\hline \hline$k_{m}$ & even $L$ & $\nu$ & $k_{m}$ & odd $L$ & $\nu$ \\
\hline 11 & 10 & 0.3459 & 11 & 11 & 0.3144 \\
18 & 12 & 0.3474 & 18 & 13 & 0.3207 \\
29 & 14 & 0.3469 & 29 & 15 & 0.3238 \\
47 & 16 & 0.3471 & 47 & 17 & 0.3267 \\
\hline \hline
\end{tabular}

odd $L=\alpha+1$ have the same maximum in-degree $k_{m}$. From these results one can see clearly that for even $L k_{m}$ follows scaling law with $\nu \simeq 0.345$. But for odd $L$ there is no scaling behavior for $k_{m}$. The same conclusion also appears in R77 which is presentated in Table V.

Rule 77 is treated in a different way. Fig. 9 is the evolution according to $\mathbf{R} 77$. The network configurations are completely different for $L$ being odd or even. For illustration it is shown in Fig. 10 the networks for $L=4$ and 5. Hence one would expect $k_{m}$ varies with $L$. The hubstates of the $\mathbf{R} 77$ are $(0101 \ldots 0101)$ and $(1010 \ldots 1010)$ when $L$ is even, but $k_{m}$ of odd $L$ is quite varying, as given in Table $\mathrm{V}$. For R77, where $L=10$ we observed that there exist only two hubstates where 22 hubstates are found for $L=11$. Concentrating on one of $L=10$ hubstates, it is clear that the hubstate of $L=11$ is just adding 1 or 0 to the end of $L=10$ 's hubstate. Furthermore, there are totally 46 preimages for $L=10$ and only 28 for $L=11$. The 18 italic configurations with $L=10$ do not associate with the preimages in $L=11$. From Fig. 11 it is known that only those preimages of $L=10$ without (111) at the boundary for can associate to the preimages of $L=11$.

To evaluate the $k_{m}$ with odd $L$ we follow the approach suggested in [3] in obtaining arbitrary in-degree of $\mathbf{R} 4$, where apart from the $\widetilde{\mathbf{T}}$ matrix itself an auxiliary matrix is constructed to resolve the difficulty. Therefore, by this approach we propose the following formula:

$$
k\left(S_{L=o d d}\right)=\operatorname{Tr}\left[\widetilde{\mathbf{T}}_{77}^{L-1} \times \widetilde{T}_{\text {multiplied }}\right] .
$$

This is due to the fact that the hubstates of odd $L$ can be viewed as just adding "0" or " 1 " in the hubstates of even L. According to Fig. 10, we could assign the $\widetilde{T}_{\text {multiplied }}$, which just corresponds to adding " 0 " in the hubstate obtained by referring to Fig. 11] since the mapping at the boundary of preimages with $L=11$ has only two

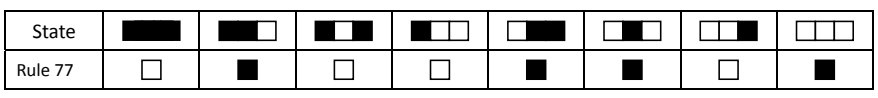

FIG. 9. The diagram of $\mathbf{R} 77$.
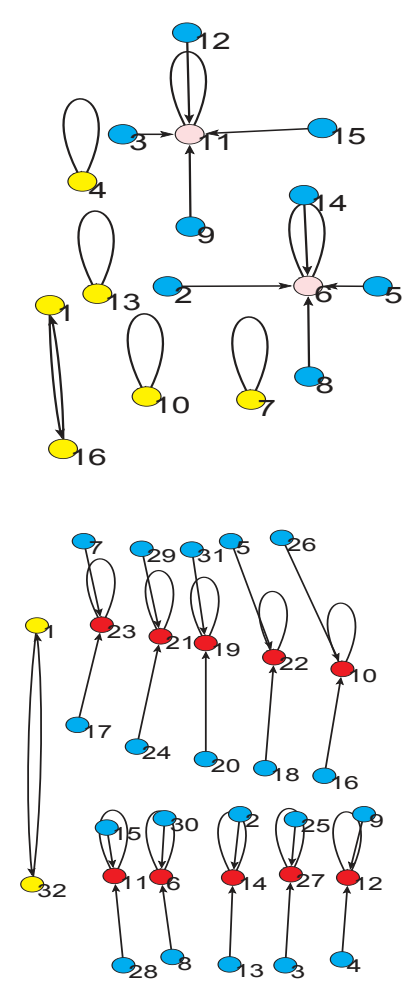

FIG. 10. The networks of R77 with (a) $L=4$ and (b) $L=5$ which are drawn by PAJEK.

TABLE V. Maximum in-degree corresponding to lattice length $L$ in $\mathbf{R} 77$ by direct counting.

\begin{tabular}{ccc|ccc}
\hline \hline$k_{m}$ & even $L$ & $\nu$ & $k_{m}$ & odd $L$ & $\nu$ \\
\hline 46 & 10 & 0.552356 & 28 & 11 & 0.437032 \\
98 & 12 & 0.551226 & 60 & 13 & 0.454376 \\
211 & 14 & 0.551507 & 129 & 15 & 0.467415 \\
453 & 16 & 0.55146 & 277 & 17 & 0.477279 \\
\hline \hline
\end{tabular}

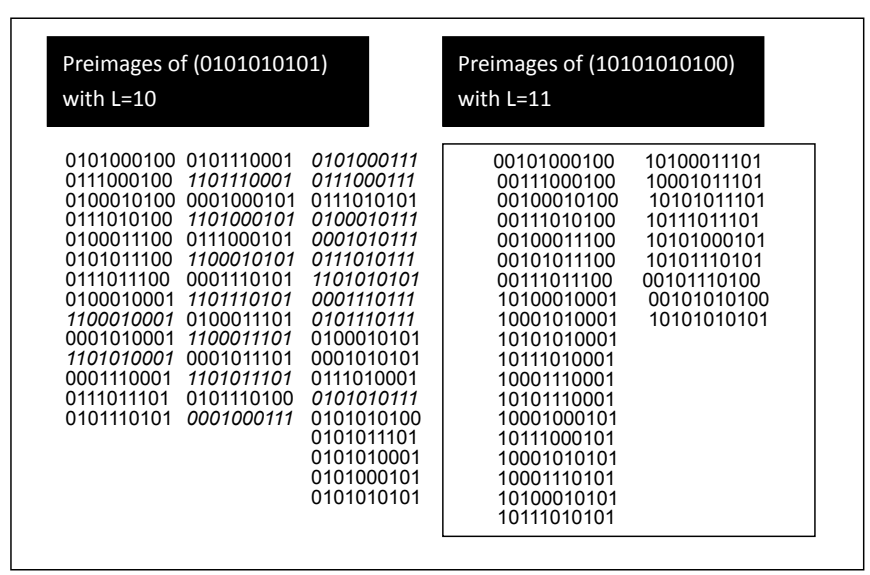

FIG. 11. The lists of pre-images of particular hubstates in R77 with different $L$. 
TABLE VI. Summary for maximum in-degree calculation for R50 and R77.

\begin{tabular}{ll}
\hline \hline Rules & Method \\
\hline $\mathbf{R} 50$ & $k\left(S_{L=\text { even }}\right)=\frac{1}{2} \operatorname{Tr}\left[\widetilde{\mathbf{T}}_{50}^{L}\right]$ \\
& $k\left(S_{L=\text { odd }}\right)=\frac{1}{2} \operatorname{Tr}\left[\widetilde{\mathbf{T}}_{50}^{L-1}\right]$ \\
\hline $\mathbf{R} 77$ & $k\left(S_{L=\text { odd }}\right)=\frac{1}{2} \operatorname{Tr}\left[\widetilde{\mathbf{T}}_{77}^{L}\right]$ \\
& $k\left(S_{L=\text { odd }}\right)=\operatorname{Tr}\left[\widetilde{\mathbf{T}}_{77}^{L-1} \times \widetilde{T}_{\text {multiplied }}\right]$ \\
\hline
\end{tabular}

possibilities:

$$
\begin{aligned}
& \widetilde{T}_{\text {multiplied }}|000>=| 000>. \\
& \widetilde{T}_{\text {multiplied }}|011>=| 110>.
\end{aligned}
$$

Therefore we have $\widetilde{T}_{\text {multiplied }}$ as

$$
\widetilde{T}_{\text {multiplied }}=\left(\begin{array}{cccccccc}
1 & 0 & 0 & 0 & 0 & 0 & 0 & 0 \\
0 & 0 & 0 & 0 & 0 & 0 & 0 & 0 \\
0 & 0 & 0 & 0 & 0 & 0 & 0 & 0 \\
0 & 0 & 0 & 0 & 0 & 0 & 1 & 0 \\
0 & 0 & 0 & 0 & 0 & 0 & 0 & 0 \\
0 & 0 & 0 & 0 & 0 & 0 & 0 & 0 \\
0 & 0 & 0 & 0 & 0 & 0 & 0 & 0 \\
0 & 0 & 0 & 0 & 0 & 0 & 0 & 0
\end{array}\right) .
$$

Then we can get the exact maximum in-degree of hubstate with any lattice length $L$. This analytical results agree with direct counting for $L \leq 25$. A summary of the formulas for the maximum in-degree of $\mathbf{R} 50$ and $\mathbf{R} 77$ are given in Table VI.

In this section we have found more rules which could not be treated with $4 \times 4 \mathbf{T}$ matrix can now be dealt with by $8 \times 8$ transfer matrix. Consequently, the maximum indegree and the ID can be evaluated efficiently.

\section{CONCLUSION}

A detail discussion of the local characteristic of network generated by elementary CA is presented in this work. First we have located the possible source of errors in claiming the multiscaling of such network in [3]. This is due to a misused of derivative on large difference. Secondly the in-degree distributions of many rules were calculated by direct counting method and the multiscaling characteristic does not appear. From the studies up to $L=25$, it suggests that the in-degree distribution of $\mathrm{CA}$ is $L$ independent regardless of its classes. Furthermore all the possible largest eigenvalues $\lambda_{m}$ for $4 \times 4$ transfer matrix $\mathbf{T}$ are obtained and analysis on the correlation between $\nu=\log _{2} \lambda_{m}$ and classification of $\mathrm{CA}$ is discussed. We have found that there might be more rules sharing the same in-degree distribution but corresponding to different classification according to $\mathrm{S}$. Wolfram. It has been found that there are good correlation for class IV which possess $\nu=0.4065$, however, apart from that, the ID of other classes do not share any universal pattern and also the value of $\nu$ can not be used as an indicator for classification. Therefore the in-degree distribution is not a proper characteristic to classification which was also pointed out in 3]. In section III the transfer matrix was extended to $8 \times 8 \widetilde{\mathbf{T}}$ matrix. This augmentation in some sense respects the evolution of $\mathrm{CA}$ rule and is also more natural to reflect the characters of hubstates' pre-images. As a result the $8 \times 8$ matrix scheme was applied to several rules which do not possess a $4 \times 4 \mathbf{T}$ matrix. The $32 \times 32$ T matrix has also applied to $\mathrm{CA}$ beyond neighbor evolution. The $L$ dependence of the hubstates of some particular rules such as $\mathbf{R} 50$ and $\mathbf{R} 77$ are discussed in detail. The calculation of their $k_{m}$ are also treated in this work.

\section{ACKNOWLEDGMENTS}

This work was supported by National Science Council of Taiwan, NSC-97-2112-M-006-003-MY2.
* hellont92@gmail.com

$\dagger$ chiachu@phys.ncku.edu.tw

[1] J. von Neuman, University of Illinois, Urbana, IL (1966).

[2] S. Wolfram, Physica (Amsterdam) 10D, 1 (1984).
[3] A. Shreim et al., Phy. Rev. Lett. 98, 198701 (2007).

[4] M. E. J. Newman, SIAM Review (2003).

[5] Y. Kayama et al., Physics Letters A 198, 23-33 (1995). 